\title{
Covariance properties and regularization of conserved currents in tetrad gravity
}

\author{
Yuri N. Obukhov* \\ Instituto de Física Teórica, Universidade Estadual Paulista, Rua Pamplona 145, 01405-900 São Paulo, Brazil \\ Department of Theoretical Physics, Moscow State University, 117234 Moscow, Russia \\ Guillermo F. Rubilar ${ }^{\dagger}$ \\ Instituto de Física Teórica, Universidade Estadual Paulista, Rua Pamplona 145, 01405-900 São Paulo, Brazil \\ Departamento de Física, Universidad de Concepción, Casilla 160-C, Concepción, Chile
}

(Received 18 April 2006; published 13 June 2006)

\begin{abstract}
We discuss the properties of the gravitational energy-momentum 3-form within the tetrad formulation of general relativity theory. We derive the covariance properties of the quantities describing the energymomentum content under Lorentz transformations of the tetrad. As an application, we consider the computation of the total energy (mass) of some exact solutions of Einstein's general relativity theory which describe compact sources with asymptotically flat spacetime geometry. As it is known, depending on the choice of tetrad frame, the formal total integral for such configurations may diverge. We propose a natural regularization method which yields finite values for the total energy-momentum of the system and demonstrate how it works on a number of explicit examples.
\end{abstract}

DOI: 10.1103/PhysRevD.73.124017

PACS numbers: 04.20.Cv, 04.20.Fy, 04.50.+h

\section{INTRODUCTION}

The problem of energy-momentum of the gravitational field belongs to the oldest in modern theoretical physics. The concepts of energy and momentum are fundamental ones in classical field theory. Within the general LagrangeNoether approach, conserved currents arise from the invariance of the classical action under transformations of fields. In particular, energy and momentum are related to time and space translations. However, due to the geometric nature of the gravitational theory and because of the equivalence principle which identifies locally gravity and inertia, the definition of gravitational energy remains a problem. In general, there are no symmetries in Riemannian manifolds that can be used to generate the corresponding conserved energy-momentum currents. It is possible, though, to associate energy and momentum to asymptotically flat gravitational field configurations. The history of the problem and corresponding achievements is described in reviews [1-3], for example.

There are several approaches to the study of the gravitational energy. The traditional one is to use the metric tensor as the field variable, choose the local spacetime coordinates and construct the energy-momentum pseudotensor. Well-known examples are the Einstein [4], LandauLifshitz [5], Bergmann [6] expressions or their generalizations [7]. A nice overview can be found in [8,9]. Closely related is the Hamiltonian approach in which, after the choice of a $(1+3)$-decomposition of spacetime, one takes the spatial components of the metric together with the conjugate momenta as the basic variables $[2,10,11]$. The total energy of the asymptotically flat configurations is

\footnotetext{
*Email address: yo@ift.unesp.br

${ }^{\dagger}$ Email address: grubilar@udec.cl
}

then defined by the surface (boundary) terms in the Hamiltonian [12]. A discussion of covariant Hamiltonian formulation of general relativity and of gauge gravity models is presented in [8,9,13-15]. Of more recent developments it is worthwhile to mention the quasilocal approach in which the conserved quantities are associated to extended but finite domains of spacetime, see the extensive review and the literature in [3].

We will analyze the definition and the properties of the energy-momentum in the framework of the tetrad formulation of general relativity. This approach was started with the work of Møller [16], for the early developments see also [17-19]. More recently the interest in the tetrad formulation of gravity has been revived [20-24]. In particular, considerable efforts [25-33] have been devoted to the study of the properties of the energy-momentum densities of gravitating systems within the formalism of the socalled teleparallel equivalent of general relativity (TEGR). The latter can be formulated as a gauge theory of the spacetime translation group. The corresponding energy-momentum currents can be defined following the traditional gauge approach $[27,28]$, or from a Hamiltonian formulation [25]. These energy-momentum currents are naturally covariant under general coordinate transformations, invariant under gauge transformations, and transform covariantly under global Lorentz transformations of the coframe (tetrad field) [27]. On the other hand, they are not covariant under local Lorentz transformations. As a consequence, the corresponding energy and momentum densities and also the total conserved quantities in a given spacelike region are coordinate independent. However, they do depend on the chosen frame. It has been shown that the energy so defined agrees with the ADM energy $[2,10]$ for asymptotically flat spacetimes [26]. Furthermore, it also gives the correct Bondi energy [29]. 
All the relevant computations have required the use of tetrads with appropriate asymptotic behavior at spatial infinity. On the other hand, there have been some attempts to study the properties and proper interpretation of the conserved quantities when computed in frames related by Lorentz transformations which are not global, see [30]. These studies are restricted to some particular solutions of the gravitational field equations and to quite specific choices of frames.

One of the aims of our paper is to systematically investigate the covariance properties of the energy-momentum currents under changes of the tetrad. Accordingly, we derive the explicit transformation laws of the canonical current and of the related field momentum under finite general linear and local Lorentz transformations. Although such a computation can be done directly within the framework of the (purely) tetrad formulation, we find it more convenient to use the natural embedding of the tetrad gravity into the general scheme of the metric-affine gravity (MAG) models. The corresponding formalism was developed for the gauge approach to gravity on the basis of the general affine group [34,35] and it was applied recently to the analysis of the general teleparallel gravity models [36].

It turns out that the transformation of the field momentum and of the canonical energy-momentum are accompanied by a change of the Lagrangian. The latter is shifted by a total derivative that effectively changes the boundary conditions while leaving the field equations invariant. All the transformed energy-momentum currents are conserved like the original one, so in this way we derive a family of conserved energy-momenta parametrized by the elements of the local Lorentz group. This family is associated with a corresponding family of boundary conditions, in complete agreement with observations in [8].

From the physical point of view, a choice of a (co)frame can be interpreted as a choice of the reference system of an observer. It is obvious that the observer's own dynamics, the state and structure of the corresponding reference system, can affect the physical measurements, including the determination of the energy and momentum of the gravitational systems. A particular example of the change of the total energy-momentum for a boosted observer is discussed in [30]. The knowledge of the covariance properties of the field momentum and the energy-momentum current is crucial for understanding the behavior of the total energy-momentum under change of reference frame. We demonstrate that for reference frames which are related by asymptotically global Lorentz transformations, the total energy-momentum transforms as a 4-vector, as expected.

The computation of the total energy in the framework of the tetrad formulation has the obvious merit of general covariance. At all steps the choice of the spacetime coordinates is unimportant because of the use of exterior forms which are coordinate invariant. However, an unfortunate choice of a (co)frame may result in a formally infinite value of the total energy-momentum (this may happen even for a flat spacetime). Accordingly, a regularization is needed, in general. A particular regularization scheme was recently proposed in [37] which is based on the idea of subtracting a contribution of a suitably introduced "reference" spacetime geometry. Here we develop a different regularization recipe which is based on the relocalization of the energymomentum currents. At the same time, the regularized currents become also covariant under the transformations of (co)frames. In this sense the proposed scheme is similar to the covariant Hamiltonian approach described in $[8,9,13-15]$.

Our general notations are as in [34]. In particular, we use the Latin indices $i, j, \ldots$ for local holonomic spacetime coordinates and the Greek indices $\alpha, \beta, \ldots$ label (co)frame components. Particular frame components are denoted by hats, $\hat{0}, \hat{1}$, etc. As usual, the exterior product is denoted by $\wedge$, while the interior product of a vector $\xi$ and a $p$-form $\Psi$ is denoted by $\xi\rfloor \Psi$. The vector basis dual to the frame 1 forms $\vartheta^{\alpha}$ is denoted by $e_{\alpha}$ and they satisfy $\left.e_{\alpha}\right\rfloor \vartheta^{\beta}=\delta_{\alpha}^{\beta}$. Using local coordinates $x^{i}$, we have $\vartheta^{\alpha}=h_{i}^{\alpha} d x^{i}$ and $e_{\alpha}=$ $h_{\alpha}^{i} \partial_{i}$. We define the volume 4-form by $\eta:=\vartheta^{\hat{0}} \wedge \vartheta^{\hat{1}} \wedge$ $\vartheta^{\hat{2}} \wedge \vartheta^{\hat{3}}$. Furthermore, with the help of the interior product we define $\left.\left.\left.\eta_{\alpha}:=e_{\alpha}\right\rfloor \eta, \eta_{\alpha \beta}:=e_{\beta}\right\rfloor \eta_{\alpha}, \eta_{\alpha \beta \gamma}:=e_{\gamma}\right\rfloor \eta_{\alpha \beta}$ which are bases for 3-, 2- and 1-forms, respectively. Finally, $\left.\eta_{\alpha \beta \mu \nu}=e_{\nu}\right\rfloor \eta_{\alpha \beta \mu}$ is the Levi-Civita tensor density. The $\eta$-forms satisfy the useful identities:

$$
\begin{gathered}
\vartheta^{\beta} \wedge \eta_{\alpha}=\delta_{\alpha}^{\beta} \eta, \\
\vartheta^{\beta} \wedge \eta_{\mu \nu}=\delta_{\nu}^{\beta} \eta_{\mu}-\delta_{\mu}^{\beta} \eta_{\nu}, \\
\vartheta^{\beta} \wedge \eta_{\alpha \mu \nu}=\delta_{\alpha}^{\beta} \eta_{\mu \nu}+\delta_{\mu}^{\beta} \eta_{\nu \alpha}+\delta_{\nu}^{\beta} \eta_{\alpha \mu}, \\
\vartheta^{\beta} \wedge \eta_{\alpha \gamma \mu \nu}=\delta_{\nu}^{\beta} \eta_{\alpha \gamma \mu}-\delta_{\mu}^{\beta} \eta_{\alpha \gamma \nu}+\delta_{\gamma}^{\beta} \eta_{\alpha \mu \nu} \\
-\delta_{\alpha}^{\beta} \eta_{\gamma \mu \nu} .
\end{gathered}
$$

The line element $d s^{2}=g_{\alpha \beta} \vartheta^{\alpha} \otimes \vartheta^{\beta}$ is defined by the spacetime metric $g_{\alpha \beta}$ of signature $(+,-,-,-)$.

\section{TRANSLATIONAL GAUGE THEORY: TETRAD GRAVITY}

Einstein's general relativity (GR) theory admits a reformulation as a tetrad theory by replacing the metric with the (co)frame as the basic field variable. It is possible to interpret such a reformulation as a gauge theory of the spacetime translation group. In the usual Yang-Mills theory of internal symmetry groups, the gauge field potential arises as a 1-form with values in the corresponding Lie algebra. In complete agreement with the general scheme, the translational gauge potential is a quartet of 1-forms $B^{\alpha}=B_{i}^{\alpha} d x^{i}$, with Greek index labeling the four generators of the abelian group of the spacetime translations. In this 
sense, the theory is constructed along the same lines as electrodynamics is constructed as a gauge theory of the abelian one-dimensional group U(1). Ultimately, such an approach introduces on the spacetime a coframe 1-form $\vartheta^{\alpha}=d x^{\alpha}+B^{\alpha}$ that is invariant under local translations. From the gauge field potential $B^{\alpha}$ we define the field strength

$$
F^{\alpha}=d B^{\alpha} \equiv d \vartheta^{\alpha}
$$

In geometrical terms, this is the anholonomity object of the coframe $\vartheta^{\alpha}$.

Now, the Yang-Mills type Lagrangian 4-form for the tetrad $\vartheta^{\alpha}$ is constructed as follows as the sum of the quadratic invariants of the field strength:

$$
\tilde{V}(\vartheta, d \vartheta)=-\frac{1}{2 \kappa} F^{\alpha} \wedge \star\left({ }^{(1)} F_{\alpha}-2^{(2)} F_{\alpha}-\frac{1}{2}{ }^{(3)} F_{\alpha}\right) .
$$

Here $\kappa=8 \pi G / c^{3}$, and ${ }^{\star}$ denotes the Hodge dual of the metric $g_{\alpha \beta}$. The latter is assumed to be the flat Minkowski metric $g_{\alpha \beta}=o_{\alpha \beta}:=\operatorname{diag}(+1,-1,-1,-1)$, and it is used also to raise and lower the Greek (local frame) indices. As it is well known, we can decompose the field strength $F^{\alpha}$ into the three irreducible pieces of the field strength:

$$
\begin{aligned}
{ }^{(1)} F^{\alpha} & :=F^{\alpha}-{ }^{(2)} F^{\alpha}-{ }^{(3)} F^{\alpha}, \\
{ }^{(2)} F^{\alpha} & \left.:=\frac{1}{3} \vartheta^{\alpha} \wedge\left(e_{\beta}\right\rfloor F^{\beta}\right), \\
{ }^{(3)} F^{\alpha} & \left.:=\frac{1}{3} e^{\alpha}\right\rfloor\left(\vartheta^{\beta} \wedge F_{\beta}\right),
\end{aligned}
$$

i.e., the tensor part, the trace, and the axial trace, respectively. This Lagrangian is a particular case of the general 3parameter Lagrangian quadratic in the field strength, see [22,28,35,36] for details.

In accordance with the general Lagrange-Noether scheme [34,35] we derive from (2.2) the translational gauge field momentum 2-form and the canonical energymomentum 3-form:

$$
\begin{gathered}
\tilde{H}_{\alpha}=-\frac{\partial \tilde{V}}{\partial F^{\alpha}}=\frac{1}{\kappa}{ }^{\star}\left({ }^{(1)} F_{\alpha}-2^{(2)} F_{\alpha}-\frac{1}{2}{ }^{(3)} F_{\alpha}\right), \\
\left.\left.\tilde{E}_{\alpha}=\frac{\partial \tilde{V}}{\partial \vartheta^{\alpha}}=e_{\alpha}\right\rfloor \tilde{V}+\left(e_{\alpha}\right\rfloor F^{\beta}\right) \wedge \tilde{H}_{\beta} .
\end{gathered}
$$

Accordingly, the variation of the total Lagrangian $L=$ $\tilde{V}+L_{\text {mat }}$ with respect to the tetrad results in the gravitational field equations

$$
d \tilde{H}_{\alpha}-\tilde{E}_{\alpha}=\Sigma_{\alpha}
$$

with the canonical energy-momentum current 3-form of matter

$$
\Sigma_{\alpha}:=\frac{\delta L_{\mathrm{mat}}}{\delta \vartheta^{\alpha}}
$$

as the source.

Using (2.6) we can write the gravitational Lagrangian as

$$
\tilde{V}=-\frac{1}{2} F^{\alpha} \wedge \tilde{H}_{\alpha} .
$$

\section{A. Conserved quantities and relocalization}

The tetrad formulation allows for a straightforward definition of the conserved quantities. From (2.8) we get

$$
d\left(\tilde{E}_{\alpha}+\Sigma_{\alpha}\right)=0 .
$$

We interpret (2.11) as the conservation law of the total (gravity plus matter) energy-momentum. On the other hand, since $\tilde{E}_{\alpha}+\Sigma_{\alpha}=d \tilde{H}_{\alpha}$, the 2-form of the field momentum $\tilde{H}_{\alpha}$ plays the role of the corresponding superpotential. We then define the total 4-momentum of the system within a spacelike hypersurface $S$ as the integral

$$
\tilde{P}_{\alpha}=\int_{S}\left(\tilde{E}_{\alpha}+\Sigma_{\alpha}\right)
$$

Or, using the field equations,

$$
\tilde{P}_{\alpha}=\int_{\partial S} \tilde{H}_{\alpha}
$$

However, there is a certain freedom to define the local energy-momentum densities. The field equations allow for a relocalization of the form

$$
\begin{gathered}
\tilde{E}_{\alpha}^{\prime}=\tilde{E}_{\alpha}+d \Psi_{\alpha}, \\
\tilde{H}_{\alpha}^{\prime}=\tilde{H}_{\alpha}+\Psi_{\alpha} .
\end{gathered}
$$

Here $\Psi_{\alpha}$ is an arbitrary covector-valued 2-form (24 independent components). Obviously, $\quad d \tilde{H}_{\alpha}^{\prime}-\tilde{E}_{\alpha}^{\prime} \equiv$ $d \tilde{H}_{\alpha}-\tilde{E}_{\alpha}$.

Relocalizations can be related to boundary contributions to the gravitational action. Suppose we change the Lagrangian by a total differential: $\tilde{V}^{\prime}=\tilde{V}+d \Psi$. The second term will only contribute with a boundary integral to the action. We assume that the 3-form $\Psi$ depends only on the coframe and, possibly, on some external field $\Phi$, i.e. $\Psi=\Psi(\vartheta, \Phi)$. Then we still have a first order Lagrangian $V^{\prime}(\vartheta, d \vartheta)$, the field equations remain unchanged, but we find a modified canonical field momentum and energymomentum of the form (2.14) and (2.15), with $\Psi_{\alpha}=$ $-\frac{\partial \Psi}{\partial \vartheta^{\alpha}}$. Thus, a boundary term induces a relocalization. It is worthwhile to note, though, that not every relocalization is derived from a boundary term. An important example is the well-known relocalization of Belinfante-Rosenfeld which yields a symmetric energy-momentum current. 


\section{B. Local Lorentz transformations}

The tetrad formulation is explicitly generally covariant since the exterior forms are invariant under the change of the spacetime coordinates. However, none of the basic quantities (2.1), (2.6), and (2.7) are covariant under the local Lorentz transformations of the coframe. Their transformation laws under a change of the coframe $\vartheta^{\prime \alpha}=$ $\Lambda^{\alpha}{ }_{\beta}(x) \vartheta^{\beta}$ can be derived directly from the corresponding definitions. After a long calculation we find

$$
\begin{aligned}
\tilde{V}\left(\vartheta^{\prime}\right)= & \tilde{V}(\vartheta)-\frac{1}{2 \kappa} d\left[\left(\Lambda^{-1}\right)_{\gamma}^{\beta} d \Lambda_{\alpha}^{\gamma} \wedge \eta_{\beta}^{\alpha}\right], \\
\tilde{E}_{\alpha}^{\prime}\left(\vartheta^{\prime}\right)= & \left(\Lambda^{-1}\right)_{\alpha}^{\beta} \tilde{E}_{\beta}(\vartheta)+d\left(\Lambda^{-1}\right)^{\beta}{ }_{\alpha} \wedge \tilde{H}_{\beta} \\
& -\frac{1}{2 \kappa} d\left[\left(\Lambda^{-1}\right)^{\beta}{ }_{\alpha}\left(\Lambda^{-1}\right)^{\nu}{ }_{\gamma} d \Lambda^{\gamma}{ }_{\mu} \wedge \eta_{\beta}{ }^{\mu}{ }_{\nu}\right] .
\end{aligned}
$$

$$
\begin{aligned}
\tilde{H}_{\alpha}^{\prime}\left(\vartheta^{\prime}\right)= & \left(\Lambda^{-1}\right)^{\beta}{ }_{\alpha} \tilde{H}_{\beta}(\vartheta)-\frac{1}{2 \kappa}\left(\Lambda^{-1}\right)^{\beta}{ }_{\alpha}\left(\Lambda^{-1}\right)^{\nu}{ }_{\gamma} d \Lambda^{\gamma}{ }_{\mu} \\
& \wedge \eta_{\beta}{ }^{\mu}{ }_{\nu} .
\end{aligned}
$$

We will not give a "brute force" proof here, but instead we will provide a simple demonstration using the framework of metric-affine gravity in Sec. III C. To our knowledge, the explicit transformation law for the teleparallel conserved quantities was not known in the literature, and here it is computed for the first time.

Using (2.17) and (2.18) we can now easily verify that the left-hand side of the field Eqs. (2.8) is covariant: $d \tilde{H}_{\alpha}^{\prime}-$ $\tilde{E}_{\alpha}^{\prime}=\left(\Lambda^{-1}\right)_{\alpha}^{\beta}\left(d \tilde{H}_{\beta}-\tilde{E}_{\beta}\right)$. Taking into account the covariance of the canonical energy-momentum (2.9) of matter, $\Sigma_{\alpha}^{\prime}=\left(\Lambda^{-1}\right)^{\beta}{ }_{\alpha} \Sigma_{\beta}$, we thus prove that the field Eqs. (2.8) are covariant under local Lorentz transformations.

For the infinitesimal case $\Lambda^{\beta}{ }_{\alpha}=\delta_{\alpha}^{\beta}+\varepsilon^{\beta}{ }_{\alpha}$ with $\varepsilon^{\alpha \beta}=$ $-\varepsilon^{\beta \alpha}$, we have

$$
\begin{gathered}
\delta \tilde{V}=-\frac{1}{2 \kappa} d\left[d \varepsilon^{\beta}{ }_{\alpha} \wedge \eta^{\alpha}{ }_{\beta}\right]=\frac{1}{2 \kappa} d\left[d \varepsilon^{\alpha \beta} \wedge \eta_{\alpha \beta}\right] \\
\delta \tilde{E}_{\alpha}=-\varepsilon^{\beta}{ }_{\alpha} \tilde{E}_{\beta}(\vartheta)-d \varepsilon^{\beta}{ }_{\alpha} \wedge \tilde{H}_{\beta} \\
\quad+\frac{1}{2 \kappa} d\left[d \varepsilon^{\beta \gamma} \wedge \eta_{\alpha \beta \gamma}\right] \\
\delta \tilde{H}_{\alpha}=-\varepsilon^{\beta}{ }_{\alpha} \tilde{H}_{\beta}(\vartheta)+\frac{1}{2 \kappa} d \varepsilon^{\beta \gamma} \wedge \eta_{\alpha \beta \gamma} .
\end{gathered}
$$

\section{Lorentz transformations of total conserved charges}

Since the field equation is covariant, both $\tilde{E}_{\alpha}+\Sigma_{\alpha}$ and $\tilde{E}_{\alpha}^{\prime}+\Sigma_{\alpha}^{\prime}$ are conserved. As a consequence, (2.18) implies that the total conserved quantity (2.12) will change to

$$
\begin{aligned}
\tilde{P}_{\alpha}^{\prime}= & \int_{\partial S}\left(\Lambda^{-1}\right)^{\beta}{ }_{\alpha} \tilde{H}_{\beta}-\frac{1}{2 \kappa} \int_{\partial S}\left(\Lambda^{-1}\right)^{\beta}{ }_{\alpha}\left(\Lambda^{-1}\right)^{\nu}{ }_{\gamma} d \Lambda^{\gamma}{ }_{\mu} \\
& \wedge \eta_{\beta}{ }^{\mu}{ }_{\nu} .
\end{aligned}
$$

Let us assume that the Lorentz transformation $\Lambda^{\beta}{ }_{\alpha}$ is asymptotically global, i.e., $d \Lambda^{\beta}{ }_{\alpha}$ vanishes at the boundary $\partial S$, then

$$
\tilde{P}_{\alpha}^{\prime}=\left(\bar{\Lambda}^{-1}\right)^{\beta}{ }_{\alpha} \int_{\partial S} \tilde{H}_{\beta}=\left(\bar{\Lambda}^{-1}\right)^{\beta}{ }_{\alpha} \tilde{P}_{\beta},
$$

where $\bar{\Lambda}=\left.\Lambda\right|_{\partial S}$. Thus, the total energy-momentum transforms as a vector under these Lorentz transformations.

\section{Equivalence with general relativity}

The resulting theory is equivalent to Einstein's GR. In order to verify this, we first need to recover the Riemannian connection and the corresponding Riemannian curvature. Although at the beginning we did not assume any connection structure on the spacetime manifold, it can be defined from the tetrad field. Indeed, given the coframe $\vartheta^{\alpha}$ together with its field strength $F^{\alpha}=d \vartheta^{\alpha}$, we construct the 1 -form

$$
\left.\left.\left.\left.\tilde{\Gamma}_{\alpha \beta}:=\frac{1}{2}\left[e_{\alpha}\right\rfloor F_{\beta}-e_{\beta}\right\rfloor F_{\alpha}-\left(e_{\alpha}\right\rfloor e_{\beta}\right\rfloor F_{\gamma}\right) \wedge \vartheta^{\gamma}\right] .
$$

Direct inspection shows that this is a Lorentz connection. Under a local Lorentz transformation of the coframe $\vartheta^{\prime \alpha}=$ $\Lambda_{\beta}^{\alpha} \vartheta^{\beta}$ it transforms as $\tilde{\Gamma}_{\alpha}^{\prime}=\left(\Lambda^{-1}\right)^{\mu}{ }_{\alpha} \Gamma_{\mu}{ }^{\nu} \Lambda^{\beta}{ }_{\nu}+$ $\Lambda^{\beta}{ }_{\gamma} d\left(\Lambda^{-1}\right)^{\gamma}{ }_{\alpha}$. Moreover, its torsion (covariant derivative of the coframe) is zero,

$$
\tilde{D} \vartheta^{\alpha}=d \vartheta^{\alpha}+\tilde{\Gamma}_{\beta}^{\alpha} \wedge \vartheta^{\beta} \equiv 0,
$$

whereas the metric is covariantly constant $\tilde{D} g_{\alpha \beta}=$ $-\tilde{\Gamma}_{\alpha}^{\gamma} g_{\gamma \beta}-\tilde{\Gamma}_{\beta}^{\gamma} g_{\alpha \gamma}=0$ in view of the skew symmetry of (2.24). Consequently, this is indeed a Riemannian connection.

We will denote all the Riemannian objects and operations by the tilde. The Riemannian curvature 2-form is, as usual, $\tilde{R}_{\alpha}{ }^{\beta}=d \tilde{\Gamma}_{\alpha}{ }^{\beta}+\tilde{\Gamma}_{\gamma}{ }^{\beta} \wedge \tilde{\Gamma}_{\alpha}^{\gamma}$.

The final proof of the equivalence of the tetrad field Eqs. (2.8) to the Einstein field equations relies on the geometric identity

$$
{ }^{\star}\left({ }^{(1)} F_{\alpha}-2^{(2)} F_{\alpha}-\frac{1}{2}{ }^{(3)} F_{\alpha}\right) \equiv \frac{1}{2} \tilde{\Gamma}^{\beta \gamma} \wedge \eta_{\alpha \beta \gamma} .
$$

One can directly verify this by making use of (2.24) and of the definitions of the irreducible parts (2.3), (2.4), and (2.5), see also $[36,38,39]$. Accordingly, we find for the gravitational field momentum

$$
\tilde{H}_{\alpha}=\frac{1}{2 \kappa} \tilde{\Gamma}^{\beta \gamma} \wedge \eta_{\alpha \beta \gamma} .
$$

Using (2.25) and (2.27) in (2.10), the gravitational Lagrangian can be recast in the form 


$$
\tilde{V}=\frac{1}{2 \kappa} \tilde{\Gamma}_{\lambda}^{\mu} \wedge \tilde{\Gamma}^{\lambda \nu} \wedge \eta_{\mu \nu} .
$$

Here we used the identity (1.3). Hence,

$$
\begin{aligned}
\left.e_{\alpha}\right\rfloor \tilde{V}= & \left.\frac{1}{\kappa}\left(e_{\alpha}\right\rfloor \tilde{\Gamma}_{\lambda}^{\mu}\right) \wedge \tilde{\Gamma}^{\lambda \nu} \wedge \eta_{\mu \nu} \\
& +\frac{1}{2 \kappa} \tilde{\Gamma}_{\lambda}^{\mu} \wedge \tilde{\Gamma}^{\lambda \nu} \wedge \eta_{\alpha \mu \nu} .
\end{aligned}
$$

On the other hand, using (2.25) and (2.27), again with the help of (1.3), we find

$$
\begin{aligned}
\left.\left(e_{\alpha}\right\rfloor F^{\beta}\right) \wedge \tilde{H}_{\beta}= & \left.-\frac{1}{\kappa}\left(e_{\alpha}\right\rfloor \tilde{\Gamma}_{\lambda}^{\mu}\right) \wedge \tilde{\Gamma}^{\lambda \nu} \wedge \eta_{\mu \nu} \\
& +\frac{1}{2 \kappa} \tilde{\Gamma}_{\alpha}^{\beta} \wedge \tilde{\Gamma}^{\mu \nu} \wedge \eta_{\beta \mu \nu} .
\end{aligned}
$$

As a result, we obtain the explicit form for the gravitational energy-momentum (2.7)

$$
\tilde{E}_{\alpha}=\frac{1}{\kappa} \tilde{\Gamma}_{[\alpha}{ }^{\mu} \wedge \eta_{\beta] \mu \nu} \wedge \tilde{\Gamma}^{\beta \nu} .
$$

This is known as the Sparling 3-form [40,41].

Finally, let us compute the derivative of the gauge momentum (2.27). Explicitly, we have

$$
d \tilde{H}_{\alpha}=\frac{1}{2 \kappa}\left(d \tilde{\Gamma}^{\beta \gamma} \wedge \eta_{\alpha \beta \gamma}-\tilde{\Gamma}^{\beta \gamma} \wedge d \eta_{\alpha \beta \gamma}\right) .
$$

Since $d \eta_{\alpha \beta \gamma}=\eta_{\alpha \beta \gamma \nu} d \vartheta^{\nu}=-\eta_{\alpha \beta \gamma \nu} \tilde{\Gamma}_{\mu}{ }^{\nu} \wedge \vartheta^{\mu}$, after using (1.4), we find

$$
\begin{aligned}
d \tilde{H}_{\alpha}= & \frac{1}{2 \kappa}\left(d \tilde{\Gamma}^{\beta \gamma}+\tilde{\Gamma}_{\nu}{ }^{\beta} \wedge \tilde{\Gamma}^{\nu \gamma}\right) \wedge \eta_{\alpha \beta \gamma} \\
& +\frac{1}{\kappa} \tilde{\Gamma}_{[\alpha}{ }^{\mu} \wedge \eta_{\beta] \mu \nu} \wedge \tilde{\Gamma}^{\beta \nu} .
\end{aligned}
$$

Using (2.31) and (2.33), we can rewrite the tetrad field Eqs. (2.8) as Einstein's equation

$$
\frac{1}{2 \kappa} \tilde{R}^{\beta \gamma} \wedge \eta_{\alpha \beta \gamma}=\Sigma_{\alpha}
$$

\section{TETRAD THEORY AS A DEGENERATE METRIC-AFFINE GRAVITY}

The tetrad theory can be naturally "embedded" into the general framework of the metric-affine gravity (MAG). In MAG, the gravitational field potentials are the metric, coframe and the linear connection $g_{\alpha \beta}, \vartheta^{\alpha}, \Gamma_{\alpha}{ }^{\beta}$. The corresponding gauge field strengths are the nonmetricity 1-form $Q_{\alpha \beta}$, the torsion 2-form

$$
T^{\alpha}=d \vartheta^{\alpha}+\Gamma_{\beta}^{\alpha} \wedge \vartheta^{\beta},
$$

and the curvature 2-form $R_{\alpha}{ }^{\beta}$, respectively. Teleparallel gravity arises as a special case of MAG when we impose the constraints of vanishing curvature and nonmetricity:

$$
R_{\alpha}^{\beta}=d \Gamma_{\alpha}^{\beta}+\Gamma_{\gamma}^{\beta} \wedge \Gamma_{\alpha}^{\gamma}=0,
$$

$$
Q_{\alpha \beta}=-d g_{\alpha \beta}+\Gamma_{\alpha}^{\gamma} g_{\gamma \beta}+\Gamma_{\beta}^{\gamma} g_{\alpha \gamma}=0 .
$$

A Yang-Mills type Lagrangian can thus be constructed as a quadratic polynomial of the torsion. The latter has three irreducible pieces, defined along the same pattern as (2.3), (2.4), and (2.5). Accordingly, the general teleparallel model is based on a 3-parameter Lagrangian. This case was considered in [36]. Here, however, we confine our attention to the so-called teleparallel equivalent gravity model with the specific Lagrangian

$$
V(g, \vartheta, \Gamma)=-\frac{1}{2 \kappa} T^{\alpha} \wedge{ }^{\star}\left({ }^{(1)} T_{\alpha}-2^{(2)} T_{\alpha}-\frac{1}{2}{ }^{(3)} T_{\alpha}\right) .
$$

This Lagrangian coincides with (2.2) when the connection is trivial, $\Gamma_{\beta}{ }^{\alpha}=0$.

The MAG field equations are obtained from the variation of the total action with respect to the metric, coframe and connection. The so-called Oth equation which comes from the metric variation is identically satisfied, whereas the socalled 1st equation reads

$$
D H_{\alpha}-E_{\alpha}=\Sigma_{\alpha} .
$$

Here $D$ denotes the covariant exterior derivative, i.e., $D H_{\alpha}=d H_{\alpha}-\Gamma_{\alpha}{ }^{\beta} \wedge H_{\beta}$. The translational momentum and the canonical energy-momentum are, respectively:

$$
\begin{gathered}
H_{\alpha}=-\frac{\partial V}{\partial T^{\alpha}}=\frac{1}{\kappa}{ }^{\star}\left({ }^{(1)} T_{\alpha}-2^{(2)} T_{\alpha}-\frac{1}{2}{ }^{(3)} T_{\alpha}\right), \\
\left.\left.E_{\alpha}=\frac{\partial V}{\partial \vartheta^{\alpha}}=e_{\alpha}\right\rfloor V+\left(e_{\alpha}\right\rfloor T^{\beta}\right) \wedge H_{\beta} .
\end{gathered}
$$

\section{A. Degeneracy of the MAG teleparallel equivalent model}

The specific feature of the MAG model (3.4) is that it is degenerate in the following sense. Let us fix the metric and the coframe and make a shift of the connection. More exactly, let us consider the transformation of the gravitational variables $\left(g_{\alpha \beta}, \vartheta^{\alpha}, \Gamma_{\beta}^{\alpha}\right) \rightarrow\left(g_{\alpha \beta}^{\prime}, \vartheta^{\prime \alpha}, \Gamma_{\beta}^{\prime}\right)$ of the form

$$
g_{\alpha \beta}^{\prime}=g_{\alpha \beta}, \quad \vartheta^{\prime \alpha}=\vartheta^{\alpha}, \quad \Gamma_{\beta}^{\prime}=\Gamma_{\beta}{ }^{\alpha}+\Psi_{\beta}{ }^{\alpha} .
$$

We assume that the 1-form $\Psi_{\beta}{ }^{\alpha}$ is such that the teleparallel constraints (3.2) and (3.3) are preserved. Then from (3.3) we immediately see that $\Psi_{\beta}^{\alpha}$ is Lorentz-valued, i.e. $\Psi^{\alpha \beta}=-\Psi^{\beta \alpha}$.

Under the shift (3.8) with any $\Psi_{\beta}{ }^{\alpha}$ that preserves the teleparallel conditions, the Lagrangian (3.4) changes by a total derivative:

$$
V\left(g, \vartheta, \Gamma^{\prime}\right)=V(g, \vartheta, \Gamma)-\frac{1}{2 \kappa} d\left(\Psi^{\alpha \beta} \wedge \eta_{\alpha \beta}\right) .
$$


The proof of this fact relies on the following geometric identity between the contractions of the irreducible pieces of the torsion and of the nonmetricity [34,42]:

$$
\begin{aligned}
R_{\alpha \beta} & \wedge \eta^{\alpha \beta}+{ }^{(1)} T^{\alpha} \wedge{ }^{\star(1)} T_{\alpha}-2^{(2)} T^{\alpha} \wedge{ }^{\star(2)} T_{\alpha}-\frac{1^{2}}{}{ }^{(3)} T^{\alpha} \\
& \wedge{ }^{\star(3)} T_{\alpha}-{ }^{(2)} Q_{\alpha \beta} \wedge \vartheta^{\beta} \wedge{ }^{\star(1)} T^{\alpha}+2^{(3)} Q_{\alpha \beta} \wedge \vartheta^{\beta} \\
& \wedge{ }^{\star(2)} T^{\alpha}+2^{(4)} Q_{\alpha \beta} \wedge \vartheta^{\beta} \wedge{ }^{\star(2)} T^{\alpha}-\frac{1}{4}{ }^{(1)} Q_{\alpha \beta} \\
& { }^{\star(1)} Q^{\alpha \beta}+\frac{1}{2}{ }^{(2)} Q_{\alpha \beta} \wedge^{\star(2)} Q^{\alpha \beta}+\frac{1}{8}(3) Q_{\alpha \beta} \wedge^{\star(3)} Q^{\alpha \beta} \\
& \left.-{ }^{3}{ }^{(4)} Q_{\alpha \beta} \wedge^{\star(4)} Q^{\alpha \beta}-\left(^{(3)} Q_{\alpha \gamma} \wedge \vartheta^{\alpha}\right) \wedge{ }^{(4)} Q^{\beta \gamma} \wedge \vartheta_{\beta}\right) \\
& \equiv \tilde{R}_{\alpha \beta} \wedge \eta^{\alpha \beta}-d\left[\vartheta^{\alpha} \wedge{ }^{\star}\left(2 T_{\alpha}-Q_{\alpha \beta} \wedge \vartheta^{\beta}\right)\right] .
\end{aligned}
$$

With the help of the constraints (3.2) and (3.3), this identity recasts the Lagrangian (3.4) into

$$
\begin{aligned}
V(g, \vartheta, \Gamma)= & \frac{1}{2 \kappa} \tilde{R}_{\alpha \beta} \wedge \eta^{\alpha \beta} \\
& +\frac{1}{\kappa} d\left[\vartheta_{\alpha} \wedge^{\star}\left(d \vartheta^{\alpha}+\Gamma_{\beta}{ }^{\alpha} \wedge \vartheta^{\beta}\right)\right] .
\end{aligned}
$$

Subtracting from this the same relation written for a shifted connection $\Gamma^{\prime}=\Gamma+\Psi$, we prove (3.9) after some straightforward algebra.

The property (3.9) is only valid for the particular combination of the squares of the irreducible pieces of torsion in (3.4), and it is violated for the general 3-parameter teleparallel Lagrangians. The special feature (3.9) has an important consequence: the connection is completely undetermined in the MAG form of the teleparallel equivalent gravity. Strictly speaking, this makes the particular model (3.4) a nonviable theory within the MAG framework, since the field equations do not determine all of the dynamical variables $(g, \vartheta$ and $\Gamma)$. As a result, matter with spin (in general, with hypermomentum) cannot be consistently coupled to the gravitational field in this framework $[36,38,43]$. This degeneracy of the MAG scheme should be compared to the tetrad formulation of Sec. II that was shown to be equivalent to GR in a dynamically consistent way.

\section{B. Relation between MAG and tetrad objects}

Despite the degeneracy mentioned, it is very useful to consider the tetrad theory embedded into MAG because, in such an extended framework, the gravitational field Lagrangian 4-form $V$ is, by construction, invariant under the local linear transformations $\left(g_{\alpha \beta}, \vartheta^{\alpha}, \Gamma_{\beta}{ }^{\alpha}\right) \rightarrow$ $\left(g_{\alpha \beta}^{\prime}, \vartheta^{\prime \alpha}, \Gamma_{\beta}^{\prime}\right)$ given by

$$
g_{\alpha \beta}^{\prime}=\left(L^{-1}\right)_{\alpha}^{\mu}\left(L^{-1}\right)_{\beta}^{\nu} g_{\mu \nu},
$$

$$
\begin{aligned}
& \vartheta^{\prime \alpha}=L_{\beta}^{\alpha} \vartheta^{\beta}, \\
& \Gamma_{\alpha}^{\prime}=\left(L^{-1}\right)^{\mu}{ }_{\alpha} \Gamma_{\mu}{ }^{\nu} L^{\beta}{ }_{\nu}+L^{\beta}{ }_{\gamma} d\left(L^{-1}\right)^{\gamma}{ }_{\alpha},
\end{aligned}
$$

$L^{\alpha}{ }_{\beta}(x) \in G L(4, R)$. The constraints (3.2) and (3.3) are preserved by these transformations.
We will use this fact for deriving the covariance properties of the basic objects in the tetrad gravity. In particular, we are interested in the properties of the field momentum and the energy-momentum (2.6) and (2.7) under local linear and Lorentz transformations. Recall that field strength $F^{\alpha}=d \vartheta^{\alpha}$ is not a covariant object under these transformations. As a result, the transformation of $\tilde{H}_{\alpha}$ and $\tilde{E}_{\alpha}$ is nontrivial. But on the other hand, the MAG counterparts of the field momentum and the energy-momentum derived from the Lagrangian (3.4), namely, (3.6) and (3.7) are explicitly covariant.

This offers a simple way to compute the transformation laws of tetrad quantities of Sec. II, using the known covariant transformation properties of the fields in the MAG picture. For this we need to establish the explicit relations between the objects in MAG and tetrad framework.

To begin with, let us use the local linear transformations and bring the metric to the constant Minkowski values, $g_{\alpha \beta}=o_{\alpha \beta}$. The remaining linear transformations which leave the metric invariant, are just the local Lorentz transformations. In other words, the theory falls naturally into the geometrical framework of the Poincare gauge theory. Now we recall that the metric-compatible connection $\Gamma_{\alpha} \beta$ can be decomposed into the Riemannian and postRiemannian parts as

$$
\Gamma_{\alpha}{ }^{\beta}=\tilde{\Gamma}_{\alpha}{ }^{\beta}-K_{\alpha}{ }^{\beta} .
$$

Here $\tilde{\Gamma}_{\alpha}{ }^{\beta}$ is the purely Riemannian connection (2.24) and $K_{\alpha}{ }^{\beta}$ is the contortion which is related to the torsion via the identity

$$
T^{\alpha}=K_{\beta}^{\alpha} \wedge \vartheta^{\beta} .
$$

Then one can show that due to geometric identities [39], the gauge momentum (3.6) can be written as

$$
H_{\alpha}=\frac{1}{2 \kappa} K^{\mu \nu} \wedge \eta_{\alpha \mu \nu}
$$

As a result, the Lagrangian (3.4) is recast as

$$
V=-\frac{1}{2} T^{\alpha} \wedge H_{\alpha}=-\frac{1}{4 \kappa} T^{\alpha} \wedge K^{\mu \nu} \wedge \eta_{\alpha \mu \nu} .
$$

Using (3.14) and (3.16) we find the relation between the field momenta in MAG and tetrad pictures:

$$
H_{\alpha}=\tilde{H}_{\alpha}-\frac{1}{2 \kappa} \Gamma^{\mu \nu} \wedge \eta_{\alpha \mu \nu} .
$$

The direct computation for the energy-momentum 3-form yields

$$
E_{\alpha}=\tilde{E}_{\alpha}-\Gamma_{\alpha}{ }^{\beta} \wedge H_{\beta}-\frac{1}{2 \kappa} d\left(\Gamma^{\mu \nu} \wedge \eta_{\alpha \mu \nu}\right) .
$$

As a result, we verify that the left-hand side of the field 
Eq. (3.5) also depends on the Riemannian variables only:

$$
D H_{\alpha}-E_{\alpha}=d H_{\alpha}-\Gamma_{\alpha}^{\beta} \wedge H_{\beta}-E_{\alpha}=d \tilde{H}_{\alpha}-\tilde{E}_{\alpha} .
$$

Finally, directly form the identity (3.9) and (3.11) we read off the relation between the Lagrangians in the MAG and tetrad pictures:

$$
V=\tilde{V}-\frac{1}{2 \kappa} d\left(\Gamma^{\mu \nu} \wedge \eta_{\mu \nu}\right) .
$$

\section{Finite Lorentz transformations for the tetrad theory}

We will now find the behavior of the quantities defined in the tetrad formulation under local Lorentz transformations. The MAG formulation is, by construction, covariant under the general linear transformations (3.12) and (3.13). In the particular case when the metric components are fixed to be $g_{\alpha \beta}=o_{\alpha \beta}$, the symmetry is reduced to local Lorentz transformations of the frame $e_{\alpha}^{\prime}=\left(\Lambda^{-1}\right)^{\beta}{ }_{\alpha} e_{\beta}$, and of the coframe and connection:

$$
\begin{gathered}
\vartheta^{\prime \alpha}=\Lambda_{\beta}^{\alpha} \vartheta^{\beta}, \\
\Gamma_{\alpha}^{\prime}=\left(\Lambda^{-1}\right)^{\mu}{ }_{\alpha} \Gamma_{\mu}{ }_{\nu} \Lambda_{\nu}^{\beta}+\Lambda_{\gamma}^{\beta} d\left(\Lambda^{-1}\right)_{\alpha}^{\gamma} .
\end{gathered}
$$

In particular, the Lagrangian (3.4) is invariant,

$$
V\left(\vartheta^{\prime}, \Gamma^{\prime}\right)=V(\vartheta, \Gamma)
$$

and the gauge momentum (3.7) as well as the energymomentum 3-form (3.7) are covariant,

$$
\begin{gathered}
H^{\prime}{ }_{\alpha}\left(\vartheta^{\prime}, \Gamma^{\prime}\right)=\left(\Lambda^{-1}\right)_{\alpha}^{\beta} H_{\beta}(\vartheta, \Gamma), \\
E^{\prime}{ }_{\alpha}\left(\vartheta^{\prime}, \Gamma^{\prime}\right)=\left(\Lambda^{-1}\right)^{\beta}{ }_{\alpha} E_{\beta}(\vartheta, \Gamma),
\end{gathered}
$$

under the transformations (3.23).

We are, however, interested in the transformation properties of the tetrad formulation. Then we notice that the tetrad configuration

$$
\left\{g_{\alpha \beta}=o_{\alpha \beta}, \quad \vartheta^{\prime \alpha}=\Lambda_{\beta}^{\alpha} \vartheta^{\beta}, \quad \Gamma_{\alpha}^{\prime}=0\right\}
$$

is related by the Lorentz transformation (3.23) to the configuration

$$
\left\{g_{\alpha \beta}=o_{\alpha \beta}, \quad \vartheta^{\alpha}, \quad \Gamma_{\alpha}{ }^{\beta}=\left(\Lambda^{-1}\right)_{\gamma}^{\beta} d \Lambda_{\alpha}^{\gamma}\right\} .
$$

Consequently, using (3.19) and (3.21), we have for the tetrad objects

$$
\tilde{V}\left(\vartheta^{\prime}\right)=V\left(\vartheta^{\prime}, \Gamma^{\prime}\right), \quad \tilde{E}_{\alpha}^{\prime}\left(\vartheta^{\prime}\right)=E_{\alpha}^{\prime}\left(\vartheta^{\prime}, \Gamma^{\prime}\right) .
$$

Finally, combining this with the Eqs. (3.24), (3.25), and (3.26), and again using (3.18), (3.19), and (3.21), we find the explicit transformation of the tetrad formulation, i.e., expressions (2.16), (2.17), and (2.18).

\section{Conformal symmetry and the trace of energy-momentum}

It has been noticed [27,28,30] that the energymomentum current $\tilde{E}_{\alpha}$ is traceless. Here we show that this property can be understood as a consequence of the conformal symmetry of the corresponding MAG theory. Normally, the vanishing of the trace of the energymomentum current is related to the conformal symmetry of the model, recall the Maxwell electrodynamics and the Klein-Gordon theory of a scalar field with a conformal coupling. The same holds true for teleparallel gravity. Namely, the vanishing of the trace of the energymomentum, i.e. $\vartheta^{\alpha} \wedge E_{\alpha}=0$, is a direct consequence of the invariance of the MAG Lagrangian (3.4) under conformal transformations. The latter are recovered as the oneparameter abelian subgroup of the general linear transformations (3.12) and (3.13) defined by the diagonal matrices of the form $L_{\beta}^{\alpha}=e^{\lambda(x)} \delta_{\beta}^{\alpha}$.

Consider the infinitesimal conformal transformations of the MAG fields:

$$
\begin{gathered}
\delta g_{\alpha \beta}=-2 \lambda g_{\alpha \beta}, \quad \delta \vartheta^{\alpha}=\lambda \vartheta^{\alpha}, \\
\delta \Gamma_{\alpha}^{\beta}=-d \lambda \delta_{\alpha}^{\beta} .
\end{gathered}
$$

As a consequence,

$$
\delta T^{\alpha}=\lambda T^{\alpha} .
$$

The corresponding variation of the teleparallel Lagrangian $V=V(g, \vartheta, T)$ then can be written as

$$
\begin{gathered}
\delta V=\delta g_{\alpha \beta} \frac{\partial V}{\partial g_{\alpha \beta}}+\delta \vartheta^{\alpha} \wedge \frac{\partial V}{\partial \vartheta^{\alpha}}+\delta T^{\alpha} \wedge \frac{\partial V}{\partial T^{\alpha}} \\
=-2 \lambda g_{\alpha \beta} \frac{\partial V}{\partial g_{\alpha \beta}}+\lambda \vartheta^{\alpha} \wedge \frac{\partial V}{\partial \vartheta^{\alpha}}+\lambda T^{\alpha} \wedge \frac{\partial V}{\partial T^{\alpha}} \\
=\lambda\left[-2 g_{\alpha \beta} \frac{\partial V}{\partial g_{\alpha \beta}}+\vartheta^{\alpha} \wedge E_{\alpha}-T^{\alpha} \wedge H_{\alpha}\right] .
\end{gathered}
$$

In order to find the derivative of the Lagrangian with respect to the metric, we rewrite (3.17) as $V=$ $-\frac{1}{2} g_{\mu \nu} T^{\mu} \wedge H^{\nu}$ and notice that besides the explicit first factor, the metric also enters the Hodge duality operator used in $H^{\alpha}={ }^{\star} \phi^{\alpha}$, with $\phi^{\alpha}=\frac{1}{\kappa}\left({ }^{(1)} T^{\alpha}-2^{(2)} T^{\alpha}-\right.$ $\left.\frac{1}{2}^{(3)} T^{\alpha}\right)$, see (3.6). As a result, the derivative reads:

$$
\frac{\partial V}{\partial g_{\alpha \beta}}=-\frac{1}{2} T^{(\alpha} \wedge H^{\beta)}-\frac{1}{2} g_{\mu \nu} T^{\mu} \wedge \frac{\partial H^{\nu}}{\partial g_{\alpha \beta}} .
$$

We can calculate the last term with the help of the "master formula" derived in [44] for the variation of Hodge duals of arbitrary forms. From Eq. (33) of [44] we have the identity

$$
\left.\frac{\partial\left(^{\star} \phi\right)}{\partial g_{\alpha \beta}}=\vartheta^{(\alpha} \wedge\left(e^{\beta)}\right\rfloor^{\star} \phi\right)-\frac{1}{2} g^{\alpha \beta \star} \phi,
$$


for any metric-independent $p$-form $\phi$. Hence

$$
\left.\frac{\partial H^{\nu}}{\partial g_{\alpha \beta}}=\vartheta^{(\alpha} \wedge\left(e^{\beta)}\right\rfloor H^{\nu}\right)-\frac{1}{2} g^{\alpha \beta} H^{\nu} .
$$

Substituting (3.37) in (3.35) and then in (3.34), we obtain

$$
\begin{gathered}
\delta V=\lambda\left[T^{\alpha} \wedge H_{\alpha}+\vartheta^{\alpha} \wedge E_{\alpha}-T^{\alpha} \wedge H_{\alpha}\right] \\
=\lambda \vartheta^{\alpha} \wedge E_{\alpha}
\end{gathered}
$$

Thus, the invariance $\delta V=0$ of the Lagrangian under the transformations (3.30) implies the vanishing of the trace of the canonical energy-momentum current $E_{\alpha}$. Moreover, using (2.10), (3.1), (3.4), (3.19), and (3.21) and some straightforward algebra, one can show that $\vartheta^{\alpha} \wedge E_{\alpha}=$ $\vartheta^{\alpha} \wedge \tilde{E}_{\alpha}$, so that also the trace of $\tilde{E}_{\alpha}$ vanishes. Similar arguments were recently presented in [28].

\section{REGULARIZATION VIA THE RELOCALIZATION}

Now we return to the tetrad formulation, in which the coframe $\vartheta^{\alpha}$ (the translational gauge potential) is the only field variable. The corresponding gauge field strength is the anholonomity 2-form $F^{\alpha}=d \vartheta^{\alpha}$. In general, as usual in classical field theory, one expects a compact-object configuration to have trivial asymptotic values of the field strength at spatial infinity, and the integral conserved quantities to have finite values.

In the tetrad formulation, however, one can encounter situations when the field strength $F^{\alpha}$ and the Riemannian connection $\tilde{\Gamma}_{\beta}{ }^{\alpha}$ constructed from it, see (2.24), do not vanish at spatial infinity. This can lead to infinite values of the energy-momentum integral even for the flat spacetime geometry. Let us consider this case in more detail, taking as an example the flat Minkowski spacetime in the spherical coordinate system $(t, r, \theta, \varphi)$. Let us choose the coframe $\vartheta^{\alpha}$ as

$$
\begin{gathered}
\vartheta^{\hat{0}}=c d t, \quad \vartheta^{\hat{1}}=d r, \\
\vartheta^{\hat{2}}=r d \theta, \quad \vartheta^{\hat{3}}=r \sin \theta d \varphi .
\end{gathered}
$$

The field strength $F^{\alpha}=d \vartheta^{\alpha}$ is nontrivial everywhere, also at the spatial infinity $(t=$ const, $r \rightarrow \infty)$. The nonvanishing components of the corresponding Riemannian connection read

$$
\tilde{\Gamma}_{\hat{1}}^{\hat{2}}=d \theta, \quad \tilde{\Gamma}_{\hat{1}}^{\hat{3}}=\sin \theta d \varphi, \quad \tilde{\Gamma}_{\hat{2}}^{\hat{3}}=\cos \theta d \varphi
$$

Consider the 2-dimensional surface $\partial S=\{r=R, \theta, \varphi\}$ as a spatial boundary. Then we find for the total energy at a fixed time

$$
\tilde{P}_{\hat{0}}=\int_{\partial S} \tilde{H}_{\hat{0}}=-\frac{2 R}{\kappa} \int_{\partial S} \sin \theta d \theta \wedge d \varphi=-\frac{8 \pi R}{\kappa},
$$

which diverges in the limit of $R \rightarrow \infty$.

However, let us recall that the energy-momentum current and the field momentum (which plays the role of a superpotential) are defined up to relocalizations. In the present case, $\tilde{H}_{\alpha}$ diverges at spatial infinity while the components of the Riemannian connection are finite in this limit. We then "regularize" the total energy by performing a relocalization with $\Psi_{\alpha}=-\frac{1}{2 \kappa} \bar{\Gamma}^{\mu \nu} \wedge \eta_{\alpha \mu \nu}$, as described in Sec. II A. The relocalized momentum then is finite at spatial infinity and the total energy-momentum vanishes, as expected.

Now we are in a position to formulate a general recipe to find finite conserved quantities for arbitrary orthonormal frames describing nontrivial spacetime geometries. As a first step, we check whether the coframe $\vartheta^{\alpha}$ is asymptotically holonomic or not. In the latter case, the field strength $F^{\alpha}$ has a nontrivial limit $\bar{F}^{\alpha}:=\left.F^{\alpha}\right|_{\partial S}$. Next, we find the limit of the Riemannian connection of the frame $\vartheta^{\alpha}$ at spatial infinity,

$$
\bar{\Gamma}_{\alpha}^{\beta}:=\left.\tilde{\Gamma}_{\alpha}^{\beta}\right|_{\partial S}
$$

In case this limit is nontrivial, we use this connection (defined in the interior domain by a continuous extension from the boundary) to perform a relocalization along the lines of Sec. II A with the boundary term defined by the 3form $\Psi=\frac{1}{2 \kappa} \bar{\Gamma}^{\mu \nu} \wedge \eta_{\mu \nu}$. The latter depends on the original tetrad and on the connection $\bar{\Gamma}_{\alpha}{ }^{\beta}$ (which plays the role of the external field $\Phi$ ). Then $\frac{\partial \Psi}{\partial \vartheta^{\alpha}}=\frac{1}{2 \kappa} \bar{\Gamma}^{\mu \nu} \wedge \eta_{\alpha \mu \nu}$ and the relocalized momentum reads

$$
\hat{H}_{\alpha}=\tilde{H}_{\alpha}-\frac{1}{2 \kappa} \bar{\Gamma}^{\mu \nu} \wedge \eta_{\alpha \mu \nu}=\frac{1}{2 \kappa} \Delta \Gamma^{\mu \nu} \wedge \eta_{\alpha \mu \nu}
$$

were $\Delta \Gamma^{\mu \nu}:=\tilde{\Gamma}^{\mu \nu}-\bar{\Gamma}^{\mu \nu}$. This new momentum is similar to the "improved" covariant superpotentials considered in [13]. The new conserved quantities,

$$
\hat{P}_{\alpha}=\int_{\partial \Sigma} \hat{H}_{\alpha},
$$

are similar to the "regularized" energy-momentum expressions proposed in [37]. There are, however, important differences between the two regularization schemes. The regularization in [37] is defined as a subtraction of a term constructed from a tetrad that describes a "background spacetime," different from the spacetime geometry of the original system. In contrast, in our formalism the original tetrad remains the only frame, no "reference frame" is introduced, but instead a Lorentz connection shows up as an additional structure.

In a sense, the tetrad theory is effectively extended to the MAG framework. In particular, one can straightforwardly verify that the regularized Lagrangian is invariant under 
local Lorentz transformations, whereas the regularized momentum is covariant.

The connection $\bar{\Gamma}^{\mu \nu}$ which we use for regularization, is not unique. Its role is to "kill" the nontrivial (sometimes divergent) behavior of the field momentum $\tilde{H}_{\alpha}$ at spatial infinity, providing a better spatial asymptotics of the regularized $\hat{H}_{\alpha}$. In other words, the crucial condition is that $\Delta \Gamma^{\mu \nu}$ must vanish at spatial infinity. This still leaves some freedom to choose $\bar{\Gamma}^{\mu \nu}$, as we will illustrate with examples below. Different choices or $\bar{\Gamma}^{\mu \nu}$ will lead to different total conserved quantities, in general.

As a final remark, it is worthwhile to note that one can further use the resulting effective MAG scheme as follows. We consider flat Lorentz connections $\bar{\Gamma}$ (teleparallel structure), hence a local Lorentz matrix $\Lambda$ exists such that $\bar{\Gamma}=$ $\Lambda^{-1} d \Lambda$. We then can define a new transformed coframe $\vartheta^{\prime}=\Lambda \vartheta$, that by construction does not require "regularization." Such a regular tetrad is obviously not unique, since $\Lambda$ is determined up to a global Lorentz matrix factor.

\section{A. Example 1: Kerr-Newman solution}

Let us now apply our method to some explicit computations of the total energy-momentum of asymptotically flat configurations. In the first example, we consider the Kerr-Newman solution. We choose the spherical (BoyerLindquist) local coordinate system $(t, r, \theta, \varphi)$, and write the coframe as $[45,46]$

$$
\begin{gathered}
\vartheta^{\hat{0}}=\sqrt{\frac{\Delta}{\Sigma}}\left[c d t-a \sin ^{2} \theta d \varphi\right], \\
\vartheta^{\hat{1}}=\sqrt{\frac{\Sigma}{\Delta}} d r, \\
\vartheta^{\hat{2}}=\sqrt{\Sigma} d \theta, \\
\vartheta^{\hat{3}}=\frac{\sin \theta}{\sqrt{\Sigma}}\left[-a c d t+\left(r^{2}+a^{2}\right) d \varphi\right] .
\end{gathered}
$$

Here the functions and constants are defined by

$$
\begin{gathered}
\Delta:=r^{2}+a^{2}-2 m r+q^{2}, \\
\Sigma:=r^{2}+a^{2} \cos ^{2} \theta, \\
m:=\frac{G M}{c^{2}}, \quad q^{2}:=\frac{G Q^{2}}{4 \pi \varepsilon_{0} c^{4}} .
\end{gathered}
$$

In accordance with our scheme, we now have to compute the field strength $F^{\alpha}$, the Riemannian connection $\tilde{\Gamma}_{\alpha}{ }^{\beta}$ and the momentum $\tilde{H}_{\alpha}$. Since we need these quantities only asymptotically, we will express our results as a power series of $\frac{1}{r}$. We obtain the asymptotic behavior of the coframe components:

$$
\begin{gathered}
\vartheta^{\hat{0}}=\left(1-\frac{m}{r}\right) c d t-a \sin ^{2} \theta\left(1-\frac{m}{r}\right) d \varphi+\cdots \\
\vartheta^{\hat{1}}=\left(1+\frac{m}{r}\right) d r+\cdots \\
\vartheta^{\hat{2}}=\left(r+\frac{a^{2} \cos ^{2} \theta}{2 r}\right) d \theta+\cdots \\
\vartheta^{\hat{3}}=-\frac{a \sin \theta}{r} c d t+\left[r+\frac{a^{2}}{2 r}\left(1+\sin ^{2} \theta\right)\right] \sin \theta d \varphi+\cdots
\end{gathered}
$$

The asymptotic behavior of their derivatives $F^{\alpha}=d \vartheta^{\alpha}$ reads

$$
\begin{gathered}
F^{\hat{0}=}-\frac{m}{r^{2}} c d t \wedge d r-\frac{a^{2} \sin \theta \cos \theta}{r^{2}} c d t \wedge d \theta \\
-\frac{m a \sin ^{2} \theta}{r^{2}} d r \wedge d \varphi \\
-2 a\left(1-\frac{m}{r}\right) \sin \theta \cos \theta d \theta \wedge d \varphi+\cdots, \\
F^{\hat{1}}=\frac{a^{2} \sin \theta \cos \theta}{r^{2}} d r \wedge d \theta+\cdots, \\
F^{\hat{2}}=\left(1-\frac{a^{2} \cos ^{2} \theta}{r^{2}}\right) d r \wedge d \theta+\cdots, \\
F^{\hat{3}=-} \frac{a \sin \theta}{r^{2}} c d t \wedge d r+\frac{a \cos \theta}{r} c d t \wedge d \theta \\
+\sin \theta d r \wedge d \varphi+\left[r+\frac{a^{2}}{2 r}\left(1+3 \sin ^{2} \theta\right)\right] \cos \theta d \theta \\
\wedge d \varphi+\cdots
\end{gathered}
$$

The dots denote terms of higher order in $\frac{1}{r}$. Similarly, we find the asymptotic connection

$$
\begin{gathered}
\tilde{\Gamma}^{\hat{0} \hat{1}}=\left(\frac{m}{r^{2}}+\cdots\right) c d t+\left(-\frac{a \sin ^{2} \theta}{r}+\cdots\right) d \varphi, \\
\tilde{\Gamma}^{\hat{0} \hat{2}}=\left(-\frac{a \sin \theta \cos \theta}{r}+\cdots\right) d \varphi \\
\tilde{\Gamma}^{\hat{0} \hat{3}}=\left(-\frac{a \sin \theta}{r^{2}}+\cdots\right) d r+\left(\frac{a \cos \theta}{r}+\cdots\right) d \theta,
\end{gathered}
$$

$$
\tilde{\Gamma}^{\hat{1} \hat{2}}=\left(-\frac{a^{2} \sin \theta \cos \theta}{r^{3}}+\cdots\right) d r+\left(-1+\frac{m}{r}+\cdots\right) d \theta,
$$

$$
\tilde{\Gamma}^{\hat{1} \hat{3}}=\left(-1+\frac{m}{r}+\cdots\right) \sin \theta d \varphi
$$




$$
\tilde{\Gamma}^{\hat{2} \hat{3}}=\left(\frac{2 m a}{r^{3}}+\cdots\right) \cos \theta c d t+(-1+\cdots) \cos \theta d \varphi .
$$

The resulting gravitational field momentum $\tilde{H}_{\alpha}$ is given by

$$
\begin{aligned}
\kappa \tilde{H}_{\hat{0}}= & \frac{2 m a \cos \theta}{r^{3}} c d t \wedge d r-\frac{a \sin \theta}{r} c d t \wedge d \theta \\
& +\left(1+\frac{m}{r}\right) \cos \theta d r \wedge d \varphi+(-2 r+2 m \\
& \left.-\frac{2 a^{2} \sin ^{2} \theta+q^{2}-m^{2}}{r}\right) \sin \theta d \theta \wedge d \varphi+\cdots,
\end{aligned}
$$

$$
\begin{aligned}
\kappa \tilde{H}_{\hat{1}}= & -\frac{a \sin \theta}{r} d r \wedge d \theta-\left(1-\frac{m}{r}\right) \cos \theta c d t \wedge d \varphi \\
& +\cdots, \\
\kappa \tilde{H}_{\hat{2}}= & \frac{a \cos \theta}{r} d r \wedge d \theta+\left(1-\frac{m}{r}\right) \sin \theta c d t \wedge d \varphi+\cdots,
\end{aligned}
$$

$$
\begin{aligned}
\kappa \tilde{H}_{\hat{\mathrm{Z}}}= & -\frac{a^{2} \sin \theta \cos \theta}{r^{3}} c d t \wedge d r-\left(1-\frac{m}{r}\right) c d t \wedge d \theta \\
& +\frac{a \sin \theta \cos \theta}{r} d r \wedge d \varphi+a \sin ^{2} \theta\left(-2+\frac{m}{r}\right) d \theta \\
& \wedge d \varphi+\cdots
\end{aligned}
$$

The term $-2 r \sin \theta d \theta \wedge d \varphi$ in (4.28) leads to a divergent total energy-momentum.

In order to find the regularizing connection (4.4), we can simply take limit of (4.22), (4.23), (4.24), (4.25), (4.26), and (4.27) for $r \rightarrow \infty$. This yields the result:

$$
\begin{array}{ll}
\bar{\Gamma}^{\hat{0} \alpha}=0, & \bar{\Gamma}^{\hat{1} \hat{2}}=-d \theta, \quad \bar{\Gamma}^{\hat{1} \hat{3}}=-\sin \theta d \varphi, \\
& \bar{\Gamma}^{\hat{2} \hat{3}}=-\cos \theta d \varphi .
\end{array}
$$

These components do not depend of time and radial coordinates, and we extend by continuity the same values to all the spacetime manifold. We can verify that it is indeed a flat connection, i.e. its curvature vanishes identically. We now take this flat connection and compute the relocalization term $\frac{1}{2} \bar{\Gamma}^{\mu \nu} \wedge \eta_{\alpha \mu \nu}$ :

$$
\begin{aligned}
\frac{1}{2} \bar{\Gamma}^{\mu \nu} \wedge \eta_{\hat{0} \mu \nu}= & -\frac{a \sin \theta}{r} c d t \wedge d \theta+\left(1+\frac{m}{r}\right) \cos \theta d r \\
& \wedge d \varphi-\left(2 r+\frac{a^{2}}{r}\right) \sin \theta d \theta \wedge d \varphi+\cdots,
\end{aligned}
$$

$$
\frac{1}{2} \bar{\Gamma}^{\mu \nu} \wedge \eta_{\hat{1} \mu \nu}=-\left(1-\frac{m}{r}\right) \cos \theta c d t \wedge d \varphi+\cdots,
$$

$$
\frac{1}{2} \bar{\Gamma}^{\mu \nu} \wedge \eta_{\hat{2} \mu \nu}=\left(1-\frac{m}{r}\right) \sin \theta c d t \wedge d \varphi+\cdots,
$$

$$
\begin{aligned}
\frac{1}{2} \bar{\Gamma}^{\mu \nu} \wedge \eta_{\hat{3} \mu \nu}= & -\left(1-\frac{m}{r}\right) c d t \wedge d \theta \\
& -a\left(1-\frac{m}{r}\right) \sin ^{2} \theta d \theta \wedge d \varphi+\cdots
\end{aligned}
$$

Thus, the relocalized momentum (4.5) is found to be regularized:

$$
\begin{gathered}
\hat{H}_{\hat{0}}=\frac{1}{\kappa}\left[\frac{2 m a \cos \theta}{r^{3}} c d t \wedge d r\right. \\
\left.+\left(2 m-\frac{a^{2} \sin ^{2} \theta+q^{2}-m^{2}}{r}\right) \sin \theta d \theta \wedge d \varphi+\cdots\right] \\
\hat{H}_{\hat{1}}=\frac{1}{\kappa}\left[-\frac{a \sin \theta}{r} d r \wedge d \theta+\cdots\right] \\
\hat{H}_{\hat{2}}=\frac{1}{\kappa}\left[\frac{a \cos \theta}{r} d r \wedge d \theta+\cdots\right] \\
\frac{1}{\kappa}\left[\frac{a \sin \theta \cos \theta}{r} d r \wedge\left(d \varphi-\frac{a}{r^{2}} c d t\right)\right. \\
\left.-a \sin ^{2} \theta d \theta \wedge d \varphi+\cdots\right]
\end{gathered}
$$

Finally, the corresponding conserved energy-momentum (4.6) reads

$$
\hat{P}_{\alpha}=\left(M c, 0,0,-\frac{\pi a c^{3}}{8 G}\right) .
$$

Notice that at spatial infinity, the vector frame dual to (4.7), (4.8), (4.9), and (4.10) is of the form $e_{\hat{0}}=\partial_{t}+O\left(r^{-2}\right)$, $e_{\hat{1}}=\partial_{r}+O\left(r^{-1}\right), \quad e_{\hat{2}}=\frac{1}{r} \partial_{\theta}+O\left(r^{-2}\right) \quad$ and $\quad e_{\hat{3}}=\frac{a}{r} \times$ $\sin \theta \partial_{t}+\frac{1}{r \sin \theta} \partial_{\varphi}+O\left(r^{-2}\right)$. We see that $e_{\hat{3}}$ has components along $\partial_{t}$ as well as along $\partial_{\varphi}$. As a result, $P_{\hat{3}}$ picks up a contribution that describes a "momentum along the $\varphi$ direction," proportional to the rotation parameter $a$.

\section{A different choice of connection}

As we have already mentioned, the regularizing connection is not unique. An alternative way to construct it is as follows. Take the Riemannian connection of the tetrad (4.7), (4.8), (4.9), and (4.10) and put the mass and the charge parameters equal zero, $m=0, q^{2}=0$. The result is the flat connection with the components

$$
\begin{aligned}
\bar{\Gamma}^{\hat{0} \hat{1}} & =-\frac{a^{\prime} r \sin ^{2} \theta}{r^{2}+a^{\prime 2} \cos ^{2} \theta} d \varphi, \\
\bar{\Gamma}^{\hat{0} \hat{2}} & =-\frac{a^{\prime} \sin \theta \cos \theta \sqrt{r^{2}+a^{12}}}{r^{2}+a^{\prime 2} \cos ^{2} \theta} d \varphi,
\end{aligned}
$$




$$
\begin{aligned}
& \bar{\Gamma}^{\hat{0} \hat{3}}=-\frac{a^{\prime} r \sin \theta}{\left(r^{2}+a^{\prime 2} \cos ^{2} \theta\right) \sqrt{r^{2}+a^{\prime 2}}} d r \\
&+\frac{a^{\prime} \cos \theta \sqrt{r^{2}+a^{\prime 2}}}{r^{2}+a^{\prime 2} \cos ^{2} \theta} d \theta, \\
& \bar{\Gamma}^{\hat{1} \hat{2}}=-\frac{a^{\prime 2} \sin \theta \cos \theta}{\left(r^{2}+a^{\prime 2} \cos ^{2} \theta\right) \sqrt{r^{2}+a^{\prime 2}}} d r \\
&-\frac{r \sqrt{r^{2}+a^{\prime 2}}}{r^{2}+a^{\prime 2} \cos ^{2} \theta} d \theta, \\
& \bar{\Gamma}^{\hat{1} \hat{3}}=-\frac{r \sin \theta \sqrt{r^{2}+a^{\prime 2}}}{r^{2}+a^{\prime 2} \cos ^{2} \theta} d \varphi, \\
& \bar{\Gamma}^{\hat{2} \hat{3}}=-\frac{\left(r^{2}+a^{\prime 2}\right) \cos ^{2} \theta}{r^{2}+a^{\prime 2} \cos ^{2} \theta} d \varphi .
\end{aligned}
$$

We have put the rotation parameter different from the original Kerr-Newman one, $a^{\prime} \neq a$, in order to stress the different nature of this connection. In the limit of $r \rightarrow \infty$ this connection tends to (4.32), so the condition $\left.\Delta \Gamma^{\mu \nu}\right|_{\partial S}=$ 0 is satisfied. For the new choice, the relocalization yields the regularized momentum (4.5) with the asymptotic $r \rightarrow$ $\infty$ behavior

$$
\begin{gathered}
\hat{H}_{\hat{0}}=\frac{2 m}{\kappa} \sin \theta d \theta \wedge d \varphi, \\
\hat{H}_{\hat{1}}=\hat{H}_{\hat{2}}=0, \\
\hat{H}_{\hat{3}}=\left(a^{\prime}-a\right) \sin ^{2} \theta d \theta \wedge d \varphi .
\end{gathered}
$$

Accordingly, the regularized total energy-momentum (4.6) reads

$$
\hat{P}_{\alpha}=\left(M c, 0,0, \frac{\pi c^{3}}{8 G}\left(a^{\prime}-a\right)\right) .
$$

This example demonstrates that the total conserved quantities obtained with the help of the regularization from the initially divergent momentum are sensitive to the choice of flat connection $\bar{\Gamma}$.

\section{Rotated regular coframe}

The connection (4.2) and (4.32) is flat. As a result, we can write it as $\bar{\Gamma}_{\alpha}{ }^{\beta}=\left(\Lambda^{-1}\right)^{\beta}{ }_{\gamma} d \Lambda^{\gamma}{ }_{\alpha}$ with some Lorentz matrix. With the help of the latter, we can rotate the original coframe (4.7), (4.8), (4.9), and (4.10) to a new tetrad $\vartheta^{\prime \alpha}=\Lambda_{\beta}^{\alpha} \vartheta^{\beta}$. Explicitly, this local Lorentz transformation can be chosen:

$$
\begin{gathered}
\vartheta^{\hat{\prime}}=\vartheta^{\hat{0}} \\
\vartheta^{\hat{\prime}}=\vartheta^{\hat{1}} \cos \varphi \sin \theta+\vartheta^{\hat{2}} \cos \varphi \cos \theta-\vartheta^{\hat{3}} \sin \varphi \\
\vartheta^{\hat{\prime}}=-\vartheta^{\hat{1}} \cos \theta+\vartheta^{\hat{2}} \sin \theta
\end{gathered}
$$

$$
\vartheta^{\hat{3}}=\vartheta^{\hat{1}} \sin \varphi \sin \theta+\vartheta^{\hat{2}} \sin \varphi \cos \theta+\vartheta^{\hat{3}} \cos \varphi \text {. }
$$

We can compute the conserved quantities in the new frame. First of all, we find that the Riemannian connection for this coframe has vanishing limit at spatial infinity. This means that no regularization is needed in this case, as expected. Furthermore, the limit value of the field momentum 2-form $\hat{H}_{\alpha}^{\prime}=\tilde{H}_{\alpha}^{\prime}$ at spatial infinity is given by

$$
\begin{aligned}
\hat{\bar{H}}^{\prime}{ }_{\alpha}= & \left(\frac{2 m}{\kappa} \sin \theta d \theta \wedge d \varphi, \frac{a}{\kappa} \sin ^{2} \theta \sin \varphi d \theta\right. \\
& \left.\wedge d \varphi, 0,-\frac{a}{\kappa} \sin ^{2} \theta \cos \varphi d \theta \wedge d \varphi\right) .
\end{aligned}
$$

The corresponding conserved total energy-momentum $\hat{P}_{\alpha}^{\prime}=(M c, 0,0,0)$ and hence we can interpret $M$ as the total mass of the system ( since $e_{\hat{0}}=\partial_{t}+O(1 / r)$ at spatial infinity). Additionally, we can verify that the regularized momentum at spatial infinity is indeed covariant under local Lorentz transformations at infinity. From (4.37), (4.38), (4.39), and (4.40) we find, at a spatial infinity,

$$
\hat{\bar{H}}_{\alpha}=\left(2 m \sin \theta, 0,0,-a \sin ^{2} \theta\right) \frac{1}{\kappa} d \theta \wedge d \varphi .
$$

It is then straightforward to confirm that (4.54) and (4.55) are related by $\hat{\bar{H}}_{\alpha}^{\prime}=\left(\Lambda^{-1}\right)^{\beta}{ }_{\alpha} \hat{\bar{H}}_{\alpha}$.

This example demonstrates that it is the asymptotics of the Riemannian connection 1-form at spatial infinity $\left(\bar{\Gamma}_{\alpha}{ }^{\beta}\right)$, and not of the anholonomity 2 -form $\bar{F}^{\alpha}$, that is crucial for determining the need of the regularization procedure. Indeed, for the new frame (4.50), (4.51), (4.52), and (4.53) we find the nontrivial limit of anholonomity $\bar{F}^{\alpha}=$ $(-2 \sin \theta \cos \theta d \theta \wedge d \varphi, 0,0,0)$ while, by construction, the limit of the Riemannian connection is trivial $\bar{\Gamma}_{\alpha}{ }^{\beta}=0$. Since the Riemannian curvature is asymptotically zero, the vanishing connection means that the corresponding frame is asymptotically inertial, whereas a nonvanishing Lorentz connection can be interpreted in terms of the acceleration and rotation of observers associated to that frame. Thus, we can say that the regularization procedure "corrects" the conserved quantities by removing "noninertial terms" at spatial infinity. If a frame is already asymptotically inertial (that is, such that its Riemannian connection vanishes asymptotically) then no regularization is necessary.

\section{B. Example 2: Configuration with mass, charge, and scalar field}

As another example, let us consider the exact spherically symmetric solution for the coupled system of the scalar, electromagnetic and gravitational field. We take the coframe as $[47,48]$ 


$$
\begin{aligned}
\vartheta^{\hat{0}}=\sqrt{f} c d \tau, & \vartheta^{\hat{1}}=\frac{1}{\sqrt{f}} d \rho, \\
\vartheta^{\hat{2}}=\sqrt{h} d \theta, & \vartheta^{\hat{3}}=\sqrt{h} \sin \theta d \varphi .
\end{aligned}
$$

Here the time and the radial coordinates $(\tau, \rho)$ are (for vanishing scalar field) different from the above $(t, r)$, and the functions depend on the variable $y=2 \mathrm{~m} / \rho$ only:

$$
\begin{gathered}
f:=\frac{\left(1-y^{2}\right)^{\mu}}{\left[k^{2}(1+y)^{\mu}-(1-y)^{\mu}\right]^{2}}, \\
h:=\rho^{2}\left(1-y^{2}\right)^{1-\mu}\left[k^{2}(1+y)^{\mu}-(1-y)^{\mu}\right]^{2} .
\end{gathered}
$$

The massless scalar field $\Phi$ and the electromagnetic field strength 2-form $F$ are, respectively:

$$
\begin{gathered}
\Phi=\sqrt{\frac{1-\mu^{2}}{2}} \log \left|\frac{1-y}{1+y}\right|, \\
F= \pm \frac{4 m \mu}{h} \sqrt{\frac{k^{2} c^{2}}{4 \pi \varepsilon_{0} G}} \vartheta^{\hat{1}} \wedge \vartheta^{\hat{0}} .
\end{gathered}
$$

Here $\varepsilon_{0}$ is the electric constant of the vacuum. The solution (4.56), (4.59), and (4.60) depends on three arbitrary integration constants: $\mu$ and $k^{2}$ are dimensionless and $m$ has the dimension of length. The total electric charge $Q$ of the system is obtained as the integral of the electric excitation 2 -form $\mathcal{D}$ over the boundary 2 -sphere of the spatial volume, $Q=\int_{\partial S} \mathcal{D}$. Explicitly, we find

$$
\mathcal{D}=H=\varepsilon_{0} c^{\star} F= \pm 4 m \mu \varepsilon_{0} c \sqrt{\frac{k^{2} c^{2}}{4 \pi \varepsilon_{0} G}} \sin \theta d \theta \wedge d \varphi .
$$

Accordingly, the total charge is

$$
Q= \pm 4 m \mu \sqrt{4 \pi \varepsilon_{0} c^{4} k^{2} / G} .
$$

As we see, all the three integration constants contribute to the total electric charge of the solution.

Let us now calculate the total mass of the solution. We begin with the analysis of the asymptotic behavior of the coframe. For $k^{2}>1$, we find

$$
\begin{aligned}
\vartheta^{\hat{0}}= & {\left[\frac{1}{k^{2}-1}-\frac{2 \mu m\left(1+k^{2}\right)}{\left(k^{2}-1\right)^{2} \rho}+\cdots\right] d \tau, } \\
\vartheta^{\hat{\imath}}= & {\left[\left(k^{2}-1\right)+\frac{2 \mu m\left(1+k^{2}\right)}{\rho}+\cdots\right] d \rho, } \\
\vartheta^{\hat{2}}= & {\left[\left(k^{2}-1\right) \rho+2 \mu m\left(1+k^{2}\right)\right.} \\
& \left.+\frac{2 m^{2}\left(k^{2}-1\right)\left(\mu^{2}-1\right)}{\rho}+\cdots\right] d \theta,
\end{aligned}
$$

$$
\begin{aligned}
\vartheta^{\hat{3}}= & {\left[\left(k^{2}-1\right) \rho+2 \mu m\left(1+k^{2}\right)\right.} \\
& \left.+\frac{2 m^{2}\left(k^{2}-1\right)\left(\mu^{2}-1\right)}{\rho}+\cdots\right] \sin \theta d \varphi .
\end{aligned}
$$

The asymptotic nonregularized gravitational field momentum then reads

$$
\begin{aligned}
\kappa \tilde{H}_{\hat{0}}= & {\left[\left(k^{2}-1\right)+\frac{2 \mu m\left(1+k^{2}\right)}{\rho}\right] \cos \theta d \rho \wedge d \varphi } \\
& -\left[2\left(k^{2}-1\right) \rho-\frac{4 m^{2} \mu^{2}\left(k^{2}-1\right)}{\rho}\right] \sin \theta d \theta \wedge d \varphi \\
& +\cdots, \\
\kappa \tilde{H}_{\hat{1}}= & -\left[\frac{1}{k^{2}-1}-\frac{2 \mu m\left(1+k^{2}\right)}{\left(k^{2}-1\right)^{2} \rho}\right] \cos \theta d \tau \wedge d \varphi+\cdots, \\
\kappa \tilde{H}_{\hat{2}}= & {\left[\frac{1}{k^{2}-1}-\frac{2 \mu m\left(1+k^{2}\right)}{\left(k^{2}-1\right)^{2} \rho}\right] \sin \theta d \tau \wedge d \varphi+\cdots, } \\
\kappa \tilde{H}_{\hat{\mathrm{J}}}= & -\left[\frac{1}{k^{2}-1}-\frac{2 \mu m\left(1+k^{2}\right)}{\left(k^{2}-1\right)^{2} \rho}\right] d \tau \wedge d \theta+\cdots
\end{aligned}
$$

In view of the divergent term in (4.67), regularization is needed. For the limiting connection $\bar{\Gamma}$, we again obtain (4.32). So, we can perform the regularization with the same flat connection used before. The final regularized gravitational field momentum then has the asymptotics

$$
\begin{aligned}
& \kappa \hat{H}_{\hat{0}}= {\left[4 \mu m\left(1+k^{2}\right)+\frac{4 m^{2}\left(2 \mu^{2}-1\right)\left(k^{2}-1\right)}{\rho}+\cdots\right] } \\
& \times \sin \theta d \theta \wedge d \varphi \\
& \kappa \hat{H}_{\hat{1}}=0 \\
& \kappa \hat{H}_{\hat{2}}=\frac{2 m^{2}}{\left(k^{2}-1\right) \rho^{2}} \sin \theta d \tau \wedge d \varphi+\cdots \\
& \kappa \hat{H}_{\hat{\mathcal{Z}}}=-\frac{2 m^{2}}{\left(k^{2}-1\right) \rho^{2}} d \tau \wedge d \theta+\cdots
\end{aligned}
$$

This yields, for the regularized total energy-momentum,

$$
P_{\alpha}=\left(2 \mu m c^{3}\left(1+k^{2}\right) / G, 0,0,0\right) \text {. }
$$

Consequently, the total gravitating mass is $M=$ $2 \mu m c^{2}\left(1+k^{2}\right) / G$. To put it differently, we find the combination of the parameters in terms of the total mass: $2 \mu m\left(1+k^{2}\right)=G M / c^{2}$. For the case $k^{2}<1$ all the above quantities change sign except for $\bar{\Gamma}$ that remains the same, so we obtain $M=-2 \mu m c^{2}\left(1+k^{2}\right) / G$. The requirement of positive total energy then implies that we have to choose $\mu m>0$ for $k^{2}>1$ and $\mu m<0$ for $k^{2}<1$. 


\section{COMPARING TETRAD AND METRIC FORMULATIONS}

There exists a natural relation between the tetrad formulation and the traditional metric formulation of GR. It is based on the fact that the usual theory is actually a tetrad theory but just considered with respect to the holonomic coordinate frame $d x^{i}$. Accordingly, the basic quantities in the tetrad and in the metric formulations are related by means of the linear transformation $d x^{i}=L^{i}{ }_{\alpha} \vartheta^{\alpha}$ from the anholonomic frame $\vartheta^{\alpha}$ to the holonomic frame $d x^{i}$. The components of the corresponding matrix are evidently just the coefficients of the coframe, i.e., $L^{i}{ }_{\alpha}=h_{\alpha}^{i}$.

It is straightforward to generalize the transformation formulas (2.16), (2.17), and (2.18) by extending them from the Lorentz matrices $\Lambda$ to the linear matrices $L$. For the field momentum we then find the transformation

$$
\begin{aligned}
\tilde{H}_{i}^{\prime}(d x)= & \left(L^{-1}\right)^{\alpha}{ }_{i} \tilde{H}_{\alpha}(\vartheta)-\frac{1}{2 \kappa}\left(L^{-1}\right)^{\alpha}{ }_{i}\left(L^{-1}\right)^{\nu}{ }_{j} d L^{j}{ }_{\mu} \\
& \wedge \eta_{\alpha}{ }^{\mu}{ }_{\nu} .
\end{aligned}
$$

Now let us compare the explicit components of the field momentum (2.27) in the anholonomic frame with its components in the holonomic frame. First we recall that $\eta_{\alpha \beta \gamma}=\sqrt{-g} h_{\alpha}^{i} h_{\beta}^{j} h_{\gamma}^{k} \epsilon_{i j k} \quad$ and $\quad \eta_{\alpha \beta}=\sqrt{-g} h_{\alpha}^{i} h_{\beta}^{j} \epsilon_{i j}$, where $\epsilon_{i j k}=\epsilon_{i j k l} d x^{l}$ and $\epsilon_{i j}=\frac{1}{2} \epsilon_{i j k l} d x^{k} \wedge d x^{l}$ with the numeric Levi-Civita symbol $\epsilon_{i j k l}$. Then directly from (2.27) we find

$$
\begin{aligned}
\tilde{H}_{\alpha} & =h_{\alpha}^{k} \mathcal{U}_{k}^{i j} \epsilon_{i j}, \\
\mathcal{U}_{k}^{i j} & =\frac{\sqrt{-} \bar{g}}{2 \kappa}\left(\gamma_{k}^{i j}+\delta_{k}^{i} \gamma_{l}^{j l}-\delta_{k}^{j} \gamma_{l}^{i l}\right),
\end{aligned}
$$

where $\gamma_{i j}{ }^{k}:=h_{j}^{\alpha}\left(\partial_{i} h_{\alpha}^{k}+\tilde{\Gamma}_{i l}^{k} h_{\alpha}^{l}\right)$ is defined with the help of the usual Christoffel connection $\tilde{\Gamma}_{i l}^{k}=\frac{1}{2} g^{k j}\left(\partial_{i} g_{l j}+\right.$ $\left.\partial_{l} g_{i j}-\partial_{j} g_{k l}\right)$. We thus see that the field momentum $\tilde{H}_{\alpha}$ in the anholonomic frame is expressed in terms of the Møller superpotential $\mathcal{U}_{k}^{i j}$ [16].

Now, directly from (5.1), we find the same field momentum in the holonomic frame:

$$
\begin{gathered}
\tilde{H}_{k}^{\prime}(d x)=h_{k}^{\alpha} \tilde{H}_{\alpha}(\vartheta)-\frac{1}{2 \kappa} h_{k}^{\alpha} h_{j}^{\nu} d h_{\mu}^{j} \wedge \eta_{\alpha}{ }^{\mu}{ }_{\nu} \\
=V_{k}^{i j} \epsilon_{i j}
\end{gathered}
$$

with

$$
V_{k}^{i j}=\frac{g_{k l}}{4 \kappa \sqrt{-g}} \partial_{m}\left[(-g)\left(g^{i l} g^{j m}-g^{i m} g^{j l}\right)\right] .
$$

We thus recover the components of the Freud superpotential [49] for the Einstein [4] energy-momentum.

This result shows that the well-known energymomentum complexes of Møller and of Einstein(-Freud) are actually different faces (anholonomic and holonomic, respectively) of one and the same object. This fact seems to be not noticed in the previous literature.

\section{FINAL REMARKS}

In this paper we have studied, within the tetrad formulation of GR, the covariance properties of various quantities describing the local and global energy-momentum content of gravitating systems. Our main result is given given by the formulas (2.16), (2.17), and (2.18) that describe the transformation laws of the Lagrangian, the energy-momentum current and the field momentum (the respective "superpotential") under local Lorentz transformations of a frame. The total energy-momentum does not "feel" the changes of frame inside the compact spatial region bounded by $\partial S$, but it is sensitive to the local Lorentz transformation of a frame at spatial infinity.

In general, the total conserved energy-momentum $\tilde{P}_{\alpha}$ corresponding to $\tilde{H}_{\alpha}$ does not transform covariantly under a change of frame. However, for local Lorentz transformations which become global at spatial infinity, the total energy-momentum transforms covariantly as a Lorentz vector.

When the Riemannian connection of a given frame is nontrivial at spatial infinity, the total energy-momentum can diverge and a regularization is needed. We have shown that it is possible to regularize the energy-momentum with the help of a relocalization defined by the flat connection $\bar{\Gamma}$. In addition, the regularized field momentum and canonical energy-momentum current turn out to be covariant under local Lorentz transformations. Note that relocalization of the energy-momentum currents was also recently discussed in [50,51].

It is thus possible to define global conserved quantities in any orthonormal frame, independently of any extra symmetry of spacetime (Killing vectors), cf. [13] and references therein. Alternatively, for a given vector field $\xi$ we can define a global conserved quantity by

$$
\left.Q:=\int_{\partial S}(\xi\rfloor \vartheta^{\alpha}\right) \wedge H_{\alpha}=\int_{\partial S} \xi^{\alpha} H_{\alpha}
$$

The global conserved quantity $Q$ is invariant (for any given vector field $\xi$ under both the general coordinate transformations and under local Lorentz transformations of a frame when we use the regularized field momentum $\hat{H}_{\alpha}$ above.

\section{ACKNOWLEDGMENTS}

The authors would like to thank J. G. Pereira for useful discussions and for his hospitality at IFT-UNESP. This work was supported by FAPESP (for Y.N.O.) and by CNPq (for G. F. R.). 
[1] A. Trautman, in Gravitation: An Introduction to Current Research, edited by L. Witten (John Wiley and Sons, New York, 1962), pp. 169-198.

[2] L. D. Faddeev, Usp. Fiz. Nauk 136, 435 (1982) [Sov. Phys. Usp. 25, 130 (1982)].

[3] L. B. Szabados, Living Rev. Relativity 7, 4 (2004); http:// www.livingreviews.org/irr-2004-4.

[4] A. Einstein, Ann. Phys. (Leipzig) 49, 769 (1916).

[5] L. D. Landau and E. M. Lifshitz, The Classical Theory of Fields (Addison-Wesley, Reading, MA, 1962), 2nd ed..

[6] P. G. Bergmann, Phys. Rev. 112, 287 (1958).

[7] J. N. Goldberg, Phys. Rev. 111, 315 (1958).

[8] C.-C. Chang, J. M. Nester, and C.-M. Chen, Phys. Rev. Lett. 83, 1897 (1999).

[9] J. M. Nester, Classical Quantum Gravity 21, S261 (2004).

[10] R. Arnowitt, S. Deser, and C. W. Misner, in Gravitation: An Introduction to Current Research, edited by L. Witten (John Wiley and Sons, New York, 1962), pp. 227-265.

[11] M. Blagojević, Gravitation and Gauge Symmetries (IoP, Bristol, 2002).

[12] T. Regge and C. Teitelboim, Ann. Phys. (USA) 88, 286 (1974).

[13] C.-M. Chen and J. M. Nester, Classical Quantum Gravity 16, 1279 (1999).

[14] C.-M. Chen, J. M. Nester, and R.-S. Tung, Phys. Rev. D 72, 104020 (2005).

[15] R. D. Hecht, Gen. Relativ. Gravit. 27, 537 (1995).

[16] C. Møller, Mat. Fys. Skr. Dan. Vid. Selsk. 1, No. 10, 1 (1961).

[17] C. Pellegrini and J. Plebanski, Mat. Fys. Skr. Dan. Vid. Selsk. 2, No. 4, 1 (1963).

[18] F. A. Kaempfer, Phys. Rev. 165, 1420 (1968).

[19] V. I. Rodichev, Theory of Gravity in Orthogonal Repère (Nauka, Moscow, 1974), p. 184 (in Russian).

[20] Y. M. Cho, Phys. Rev. D 14, 2521 (1976).

[21] W. Thirring and R. Wallner, Revista Brasileira de Fisica 8 , 686 (1978).

[22] K. Hayashi and T. Shirafuji, Phys. Rev. D 19, 3524 (1979).

[23] H. Meyer, Gen. Relativ. Gravit. 14, 531 (1982).

[24] U. Muench, Diploma thesis, University of Cologne, 1997.

[25] J. W. Maluf, J. Math. Phys. (N.Y.) 35, 335 (1994).

[26] J. W. Maluf and F. F. Faria, J. Math. Phys. (N.Y.) 36, 4242 (1995).

[27] V. C. de Andrade, L. C. T. Guillen, and J. G. Pereira, Phys. Rev. Lett. 84, 4533 (2000).
[28] Ya. Itin, Classical Quantum Gravity 19, 173 (2002).

[29] J. W. Maluf, Ann. Phys. (Leipzig) 13, 604 (2004).

[30] J. W. Maluf, Gravitation Cosmol. 11, 284 (2005).

[31] R. P. Wallner, Acta Phys. Austriaca 52, 121 (1980).

[32] M. Dubois-Violette and J. Madore, Commun. Math. Phys. 108, 213 (1987).

[33] F. B. Estabrook, Classical Quantum Gravity 23, 2841 (2006).

[34] F. W. Hehl, J. D. McCrea, E. W. Mielke, and Y. Ne'eman, Phys. Rep. 258, 1 (1995).

[35] F. Gronwald, Int. J. Mod. Phys. D 6, 263 (1997).

[36] Yu. N. Obukhov and J. G. Pereira, Phys. Rev. D 67, 044016 (2003).

[37] J. W. Maluf, M. V. O. Veiga, and J. F. da Rocha-Neto, grqc/0507122.

[38] Yu. N. Obukhov and J. G. Pereira, Phys. Rev. D 69, 128502 (2004).

[39] Yu. N. Obukhov, International Journal of Geometric Methods in Modern Physics 3, 95 (2006); http:// www.worldscinet.com/ijgmmp/).

[40] G. A.J. Sparling, report "Twistors, Spinors and The Einstein Equations," 1982 (to be published).

[41] G. A.J. Sparling, in Curved Twistor Spaces, Further Advances in Twistor Theory Vol. III, edited by L.J. Mason, L. P. Hughston, P.Z. Kobak, and K. Pulverer (Chapman \& Hall/CRC, Boca Raton, 2001).

[42] Yu. N. Obukhov, E. J. Vlachynsky, W. Esser, and F. W. Hehl, Phys. Rev. D 56, 7769 (1997).

[43] M. Leclerc, Phys. Rev. D 71, 027503 (2005).

[44] U. Muench, F. Gronwald, and F. W. Hehl, Gen. Relativ. Gravit. 30, 933 (1998).

[45] F. W. Hehl and Yu. N. Obukhov, Foundations of Classical Electrodynamics-Charge, Flux, and Metric (Birkhäuser, Boston, 2003).

[46] R. A. Puntigam, E. Schrüfer, and F. W. Hehl, in Computer Algebra in Science and Engineering, edited by J. Fleisher et al. (World Scientific, Singapore, 1995), pp. 195-211; gr-qc/9503023.

[47] T. Dereli and Yu. N. Obukhov, Classical Quantum Gravity 17, 219 (2000).

[48] T. Dereli and Yu. N. Obukhov, Phys. Rev. D 61, 084015 (2000).

[49] Ph. Freud, Annals of Mathematics 40, 417 (1939).

[50] L. Hannibal, J. Phys. A: Math. Gen. 29, 7669 (1996).

[51] Ya. Itin, J. Phys. A 36, 8867 (2003). 